\title{
Gêneros Euastrum Ehrenberg ex Ralfs e Micrasterias C. Agardh (Conjugatophyceae-Desmidiaceae) de dois ambientes amazônicos de águas pretas (Manaus, Amazonas-Brasil)
}

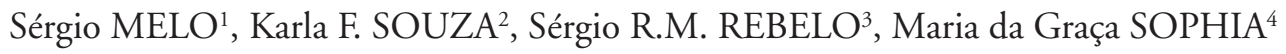 \\ RESUMO \\ Foi realizado um inventário das espécies dos gêneros Euastrum Ehrenberg ex Ralfs e Micrasterias C.Agardh de dois ambientes de \\ águas pretas do município de Manaus, um lago de inundação (lago Tupé), e o rio Negro. As coletas foram realizadas com rede \\ de plâncton em quatro estaçôes no lago e uma no rio Negro, situada próxima ao lago. Um total de 93 amostras foi analisado, \\ coletadas em escala mensal no período de março de 2002 a outubro de 2003 . As espécies foram descritas e ilustradas com base \\ na sua morfologia e morfometria. Um total de 12 espécies foi identificado, sendo cinco do gênero Euastrum (E. evolutum var. \\ perornatum; E. gemmatum var. monocyclum; E. ornans; E. sinuosum e E. spinulosum), e sete do gênero Micrasterias (M. borgei; \\ M. radiata var. brasiliensis; $M$. torreyi; $M$. laticeps var. acuminata; $M$. mahabuleshwarensis var. amazonensis; $M$. rotata var. rotata; \\ M. siolii). Todas as espécies, exceto M. radiata Hassal var. brasiliensis Grönblad, foram registradas no rio Negro, enquanto no \\ lago Tupé foi registrado, no geral, apenas sete espécies, três de Euastrum e quatro de Micrasterias.
}

PALAVRAS-CHAVE: Desmídias, Águas pretas, Lagos de inundação

\section{Genera Euastrum Ehrenberg ex Ralfs and Micrasterias C.Agardh (Conjugatophyceae-Desmidiaceae) from two Amazon black water environments (Manaus,Amazonas-Brazil)}

\begin{abstract}
An investigation of genera Euastrum Ehrenberg ex Ralfs and Micrasterias C.Agardh from an Amazon floodplain lake of the Negro river Basin was undertaken. The samples were collected using a plankton net in four stations on the Tupe Lake and one station near a lake on the Negro river. The species were identified and described on the basis of morphological and morphometrical characteristics. A total of 12 species were identified, five species of Euastrum genus (E. evolutum var. perornatum; E. gemmatum var. monocyclum; E. ornans; E. sinuosum; E. spinulosum), and seven of Micrasterias genus (M. borgei; M. radiata var. brasiliensis; M. torreyi; M. laticeps var. acuminata; $M$. mahabuleshwarensis var. amazonensis; M. rotata var. rotata; M. siolii). All species, except M. radiata Hassal var. brasiliensis Grönblad, were found in the Negro river; on the other hand, only seven species were found in the Tupé lake, three of Euastrum and four of Micrasterias.
\end{abstract}

KEYWORDS: Desmids, Black water, Floodplain lakes

\footnotetext{
1 Prof. do Programa Integrado de Pós-Graduação em Biologia Tropical e Recursos Naturais -INPA. Av. André Araújo 2936 - INPA/CPBA. CEP: 69060-0001 Aleixo - Manaus-AM. e-mail: sergio.melo@pq.cnpq.br

2 Aluna de Doutorado em Biologia de Água Doce e Pesca Interior PPG-BADPI/INPA - Bolsista FAPEAM

${ }^{3}$ Aluno de Mestrado em Ciências do Ambiente e Sustentabilidade na Amazônia -UFAM - Bolsista CAPES

${ }^{4}$ Pesquisadora do Museu Nacional-UFRJ
} 


\section{INTRODUÇÃO}

As desmídias englobam um grupo de algas da divisão Chlorophyta, classe Conjugatophyceae, ordem Desmidiales, famílias Gonatozygaceae, Peniaceae Closteriaceae e Desmidiaceae, segundo a classificação de Mix (1972), ratificada por McCourt et al. (2000) a partir de análise de biologia molecular. Este grupo de algas constitui um dos principais representantes de vários ambientes de água doce, apresentando elevada riqueza de espécies nas águas amazônicas (Melo et al. 2005a). O que é atribuído ao fato de os ambientes aquáticos amazônicos, em grande parte, apresentarem águas ácidas, com baixa condutividade elétrica e oligotróficas, condições que favorecem o sucesso das desmídias (Uherkovich, 1981; Coesel, 1982).

Embora as desmídias sejam um dos grupos de algas melhor conhecido taxonomicamente na região amazônica, ainda são relativamente poucos os ambientes estudados, se considerado a extensão territorial da Amazônia. Conseqüentemente, pouco se conhece sobre a distribuição geográfica destas algas, em especial no Estado do Amazonas onde predominam ambientes de águas pretas e de águas brancas, segundo a classificação de Sioli (1950). Informaçôes sobre trabalhos com desmídias no estado do Amazonas são sintetizadas por Lopes \& Bicudo (2003) e por Melo et al. (2004; 2005a).

Entre as desmídias, os gêneros Euastrum Ehrenberg ex Ralfs e Micrasterias C. Agardh apresentam exuberantes formas morfológicas com células geralmente planas e com variada ornamentação. Este trabalho teve por objetivo descrever e ilustrar as espécies de Euastrum e Micrasterias de dois ambientes de águas pretas do Estado do Amazonas, bem como apresentar a distribuição geográfica das espécies registradas no referido Estado.

\section{MATERIAL E MÉTODOS}

Para o presente estudo foram realizadas coletas em quatro estações no lago Tupé (ET1, ET5, ET7 e E10), um lago de inundação da margem esquerda do rio Negro, situado a cerca de $30 \mathrm{Km}$ da cidade de Manaus e em um ponto no rio Negro (EN12) localizado em frente ao lago Tupé (Figura 1). As coletas foram realizadas em escala mensal no período de março de 2002 a outubro de 2003. As amostras foram coletadas através de arrastos verticais e horizontais com rede de plâncton com abertura de malha de $25 \mu \mathrm{m}$ e fixadas em solução de Transeau (Bicudo \& Bicudo, 1970). As águas dos ambientes estudados apresentaram valores de $\mathrm{pH}$ oscilando entre 3,7 a 5,5, condutividade elétrica entre 7,9 a $23,9 \mu \mathrm{S}$ $\mathrm{cm}^{-1}$, e temperatura da água entre 28,8 a $31,8^{\circ} \mathrm{C}$ (Melo et al. 2005b).
No laboratório, as amostras foram analisadas em microscópio óptico Zeiss Oberkochen, modelo Standart 18 e Leitz Laborlux S, equipado com câmara-clara e ocular de medição. O sistema de classificação adotado foi baseado em Mix (1972), que inclui na ordem Desmidiales a família Desmidiaceae, na qual estão incluídos os gêneros Euastrum e Micrasterias.

A identificação e a descrição taxonômica das espécies foram efetuadas com base em bibliografia especializada, entre esta os trabalhos de Růžiččka (1981); Prescott (1977); Förster (1964, 1969, 1974), considerando os caracteres morfológicos, como o formato e a ornamentação, e morfométricos, tais como: comprimento da célula (C); largura máxima (L); largura do ápice ou lobo polar, istmo e razão comprimento largura.

As amostras analisadas, um total de 93, foram depositadas no Herbário do Instituto Nacional de Pesquisas da Amazônia, sob os números INPA223884 a INPA223904 e INPA223947 a INPA224018.

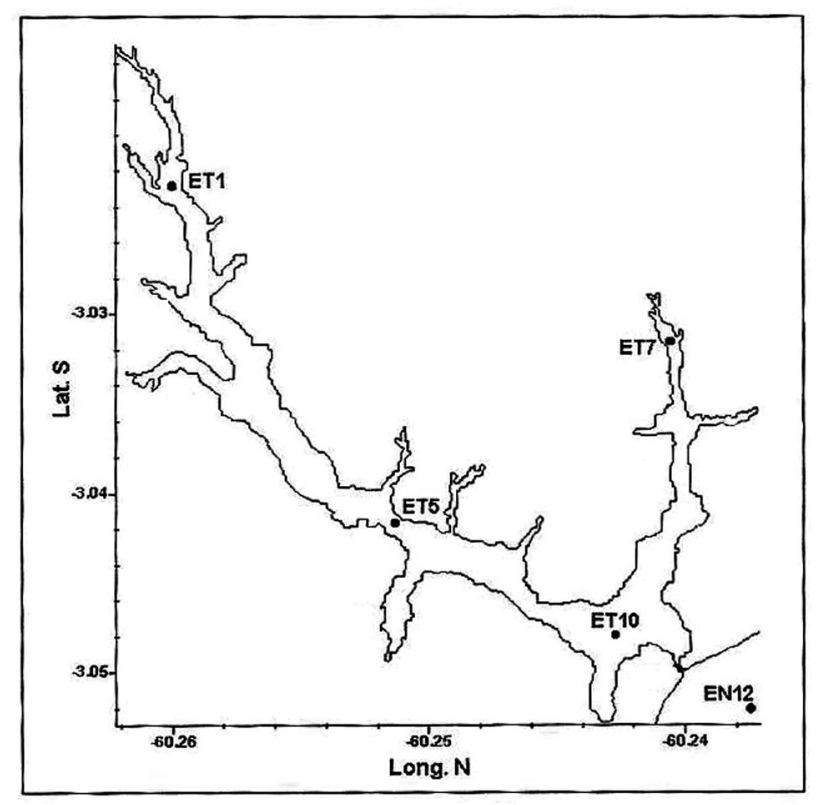

Figura 1 - Mapa do lago Tupé (Manaus, AM) com os pontos de amostragem (Fonte: Projeto Biotupé).

\section{RESULTADOS E DISCUSSÃO}

\section{CHAVE ARTIFICIAL PARA IDENTIFICAÇÃO DAS ESPÉCIES DE EUASTRUM E MICRASTERIAS}

1. Semicélulas com incisões abertas e rasas entre os lobos ..2 1. Semicélulas com incisões lineares e profundas entre os lobos ... 6

2. Parede celular lisa ............................................. 3

2. Parede celular ornamentada ..................................4 
3. Margem do lobo basal com espinho robusto ... E. ornans

3. Margem do lobo basal sem espinho ...... E. sinuosum 4.Lobo apical com espinhos divergentes E. evolutum var. perornatum

4. Lobo apical com outra ornamentação

5. Lobo apical com protuberâncias ....... E. spinulosum

5. Lobo apical com espinhos diminutos E. gemmatum var. monocyclum

6. Semicélula 3-lobada .... M. laticeps var. acuminata

6. Semicélula 5-lobada

7. Parede celular lisa ...7

7. Parede celular ornamentada

8. Lobo apical com extremidade acuminada ... M. siolii

8. Lobo apical com extremidade 2-denticulada .......9

9. Margem apical do lobo polar com entalhe mediano ... 10

9. Margem apical do lobo polar sem entalhe mediano M. radiata var. brasiliensis

10. Lobos basais e laterais retos ...... M. rotata var. rotata 10. Lobos basais e laterais curvados para cima . M. torrey var. curvata

11. Parede celular com espinhos M. borgei

11. Parede celular com protuberâncias M. mahabuleshwarensis var amazonensis

\section{DESCRIÇÃO DOS GÊNEROS E ESPÉCIES REGISTRADAS}

\section{Euastrum Ehrenberg ex Ralfs 1848}

Células solitárias, usualmente mais longas do que largas. As semicélulas são na maioria das vezes, 3- lobadas, com 2 lobos basais e um lobo polar. Em muitas espécies existem lóbulos laterais entre os lobos basais e o lobo polar. A vista lateral das células é de contorno oval raramente elíptico ou subretangular, com pólos estreitos agudos ou truncados (convexo ou reto). A parede celular pode ser lisa ou pontuada, sendo freqüentemente granulosa e com espinhos na face ou nas margens dos lobos. $\mathrm{O}$ istmo é relativamente estreito.

Euastrum evolutum var. perornatum Scott \& Croasdale in Scott, Grönblad \& Croasdale. Acta Bot. Fenn., p. 32, t. 4:74. (Figura 5)

Células cerca de 1,3-1,5 vezes mais longas que largas; semicélula semicircular-trapezoidal, seno mediano fechado; espinhos intramarginais no lobo basal; margem do lobo apical com espinhos divergentes.

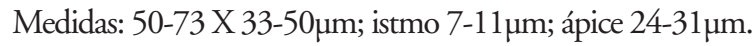

Ocorrência: ET1 (INPA 223899, INPA 223947, INPA 223995, INPA 224013), ET5 (INPA 223948, INPA 223970, INPA 223975, INPA 223979, INPA 223982, INPA 224026), ET7 (INPA 223953, INPA 223983, INPA 224015), ET10 (INPA 223866, INPA 223984, INPA 223889, INPA 223997) e EN12 (INPA 223955, INPA 223964, INPA 223978, INPA 223992).

Distribuição geográfica no Amazonas: Rio Preto da Eva (Thomasson 1971)

Euastrum gemmatum var. monocyclum Nordstedt, Acta Univ. Lund 16:8. Pl. 1, Figura 13. 1880. (Figura 2)

Células cerca de 1,1-1,2 vezes mais longas que largas; semicélula horizontalmente subretangular; seno mediano fechado; lobos basais arredondados com grânulos marginais; pólos ligeiramente retusos.

Medidas: 58-59 X 53-56 $\mu \mathrm{m}$; istmo 14-16um; ápice 19-25.

Ocorrência: EN12 (INPA 223978, INPA 223980, INPA 223985, INPA 223987).

Distribuição geográfica no Amazonas: Lago Cristalino, Lago São Sebastião (Martins, 1986), Lago Tupé e Rio Negro (Melo et al. 2005a).

Euastrum ornans Förster, Amazoniana, Vol. II (1/2), p. 35. PL.9:17-18.1969. (Figura 13)

Células cerca de 1,1-1,2 vezes mais longas que largas; semicélula trapeziforme, seno mediano linear e fechado; lobos basais com espinho, lobo apical com espinhos divergentes nos ângulos.

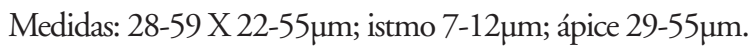

Ocorrência: ET1 (INPA 223893, INPA 223947, INPA 224013), ET5 (INPA 223884, INPA 223993), ET7 (INPA 223885, INPA 223891, INPA 223894, INPA 223949), ET10 (INPA 223886, INPA 223895, INPA 223997, INPA 224004) e EN12 (INPA 223985, INPA 223992, INPA 223994, INPA 223998).

Distribuição geográfica no Amazonas: Rio Preto da Eva (Thomasson, 1971), Lago Tupé e Rio Negro (Melo et al. 2005a).

Euastrum sinuosum Lenormand 1845, Herbarium, ex. Krieger 1937, Rabenhorst Kryptogamen-Flora 13( 1:3): 499. Pl. 62 (Figura 4)

Células cerca de 2,0-2,4 vezes mais longas que largas; semicélula truncada-oval, lobos basais arredondados; lobo apical truncado com invaginação fechada na região mediana.

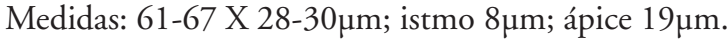

Ocorrência: ET5 (INPA 223982, INPA 223989, INPA 223993), ET7 (INPA 223953, INPA 223963, INPA 223983, INPA 224008) e EN12 (INPA 223998, INPA 224005).

Distribuição geográfica no Amazonas: Lago Tupé (Melo et al. 2005a)

Euastrum spinulosum Delponte, Mem. R. Acad. Sci. 
Di Torino, 28: 95. Pl. 6, Figuras 17, 18. 1876. (Figura 3)

Células cerca de 1,1-1,2 vezes mais longas que largas; semicélulas ovais; ângulos basais arredondados; seno mediano estreito e fechado; margem apical ligeiramente retusa na região apical, margem dos lobos com espinhos; semicélulas com uma grande protuberância circular central.

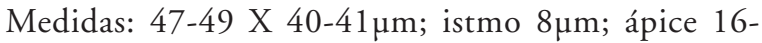
$19 \mu \mathrm{m}$.

\section{Ocorrência: EN12 (INPA 223950)}

Distribuição geográfica no Amazonas: Parque Nacional do Jaú (Melo et al. 2004)

\section{Micrasterias C.Agardh ex Ralfs 1848}

Células planas, aproximadamente circulares, possuem um istmo profundo. Cada semicélula é dividida em até cinco lobos por quatro incisões profundas que são simetricamente posicionadas sobre o eixo da célula. Os lobos se subdividem por incisões secundárias, constituindo os lóbulos. As margens das células possuem espinhos ou dentes e a parede celular pode ser lisa, verrugosa ou espinhosa. A maioria das espécies é freqüente em águas ácidas (Hoek et al. 1997).

Micrasterias borgei Krieger. Ibid. 13,1: pp. 86, Plate 128, Figuras 1-4. 1939. (Figura 7)

Células cerca de 1,1-1,4 vezes mais longas que largas; semicélula de contorno semicircular, 5- lobada. Parede celular irregularmente coberta de espinhos, incisões interlobares profundas, semiabertas, lobos basais e laterais divididos em lóbulos, 2- denticulados, lóbulos divididos em $2^{\mathrm{a}}$ e $3^{\mathrm{a}}$ ordem.

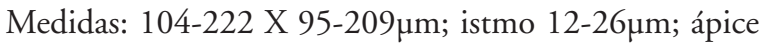
$22-49 \mu \mathrm{m}$.

Ocorrência: ET1 (INPA 223981) e EN12 (INPA 223978).

Distribuição geográfica no Amazonas: Rio Preto da Eva (Thomasson, 1971) e Parque Nacional do Jaú (Melo et al., 2004), Lago Tupé (Melo et al., 2005a) e Rio Negro (Melo et al., 2005a e Uherkovich \& Rai, 1979) .

Micrasterias laticeps Nordstedt var. acuminata Krieger Kryptogamen-Fl. Deutschl. 13(2):14; Pl.98, Figura 2.1939. (Figura 12)

Células cerca de 1,2-1,3 vezes mais larga que longa; semicélula de contorno semicircular, 3-lobada, incisóes interlobares profundas, abertas, lobo apical transversalmente fusiforme, margem apical convexa, ângulos acuminados, lobos basais semifusiformes, dispostos horizontalmente.

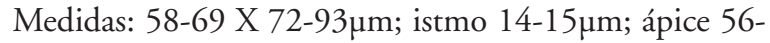
$80 \mu \mathrm{m}$.

Ocorrência: EN12 (INPA 224012).

Distribuição geográfica no Amazonas: Lago Novo Andirá (Lopes \& Bicudo, 2003).

Micrasterias mahabuleshwarensis Hobsen var. amazonensis Förster, Amazoniana, p. 40, t.17, Figura

1-8. 1969. (Figura 11)

Células cerca de 1,2 vezes mais longas que largas; semicélulas de contorno semielíptico, 5-lobadas, incisões profundas; lobo polar acentuadamente projetado além dos lobos laterais; parede celular de margem serreada; presença de espinhos intramarginais em toda a célula.

Medidas: 117 X $97 \mu \mathrm{m}$; istmo $19 \mu \mathrm{m}$; ápice $59 \mu \mathrm{m}$.

Ocorrência: EN12 (INPA 223955).

Distribuição geográfica no Amazonas: Lagos Cristalino, Lago São Sebastião (Martins, 1986), Paraná do Piranha (Uherkovich, 1981).

Micrasterias radiata Hassal var. brasiliensis Grönblad, Acta Soc. Sc. Fenn. p.15, t.4:83.1945. (Figura 10)

Células cerca de 1,1 vezes mais longas que largas; contorno subcircular, profundamente constritas na parte média, seno mediano linear, fechado na porção próxima ao istmo e aberto, acutangular distalmente; semicélulas 5-lobadas, ápice retuso, ângulos formando processos divergentes, 2- denticulados, lobos laterais e basais de tamanho iguais, sub-retangulares, cada qual subdividido em 2 lóbulos e extremidades 2denticuladas, dentículos divergentes; pólos acuminados com uma intumescência no meio de cada semicélula.

Medidas: 131 X $119 \mu \mathrm{m}$; istmo $12 \mu \mathrm{m}$; ápice $56 \mu \mathrm{m}$.

Ocorrência: ET10 (INPA 223889).

Distribuição geográfica no Amazonas: Rio Branco (Uherkovich \& Rai, 1979); Lago Tupé (Melo et al., 2005a).

Micrasterias rotata (Grev.) Ralfs var. rotata Anals Mag. nat. Hist. 14; 259, pl. 6, Figura 1. 1844. (Figura 9)

Células cerca de 1,1 vezes mais longas que largas; semicélulas 5-lobadas, incisōes lineares; lobo polar usualmente projetado além dos lobos laterais, ângulos levemente projetados, 2-denticulados; lobos basais e laterais de tamanhos desiguais; lobos laterais maiores que os basais, cada um subdividido por 3 incisōes em 4 partes iguais, 3-denticuladas.

Medidas: $131 \mathrm{X} 119 \mu \mathrm{m}$; istmo $12 \mu \mathrm{m}$; ápice $569 \mu \mathrm{m}$.

Ocorrência: ET5 (INPA 224011) e EN12 (INPA 223973, INPA 224012).

Distribuição geográfica no Amazonas: Lago Tupé (Melo et al., 2005a). 

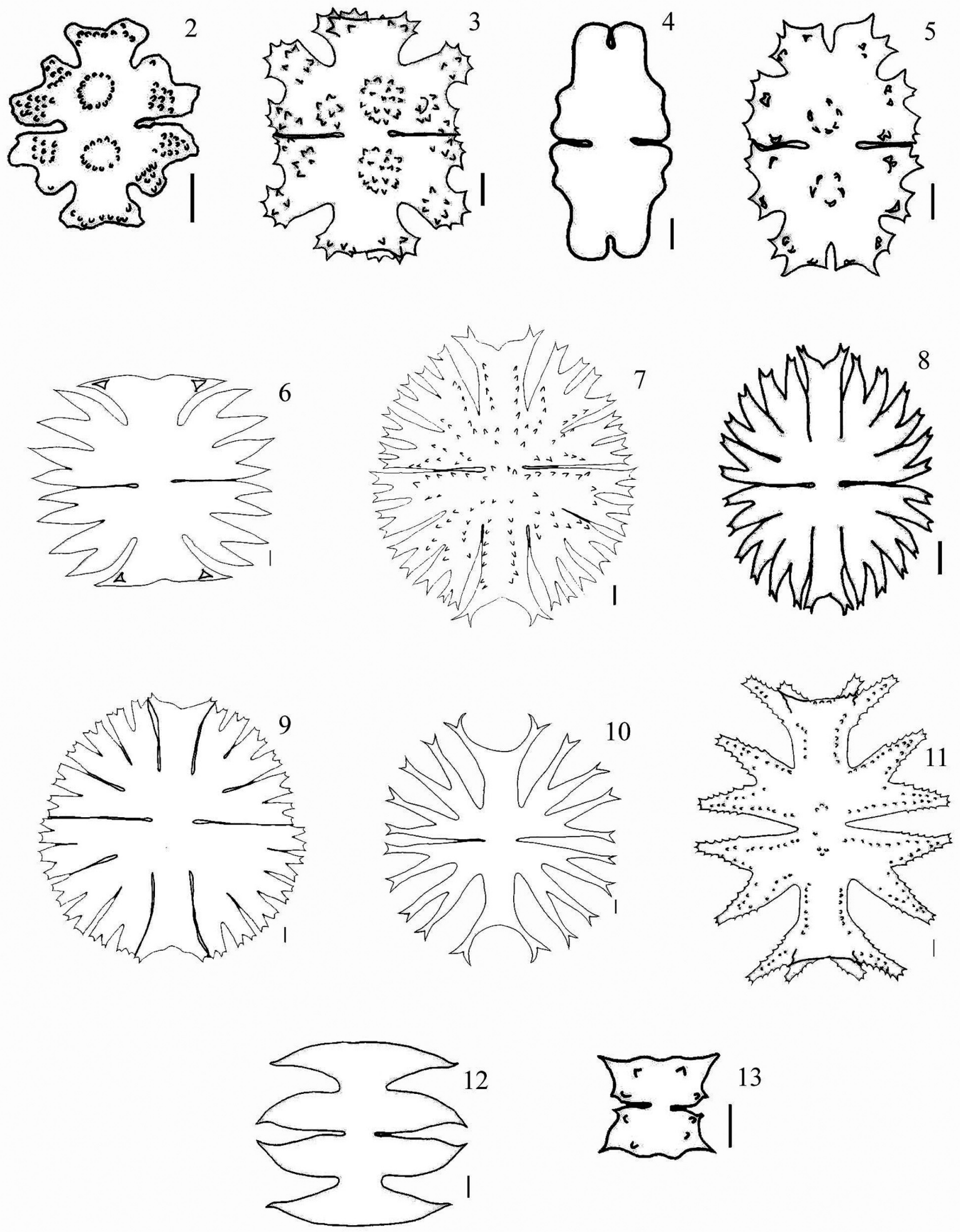

Figuras 2-13. 2 - Euastrum gemmatum var. monocyclum. 3- E. spinulosum. 4- E. sinuosum. 5- E. evolutum var. perornatum. 6- Micrasterias siolii. 7- M. borgei. 8- M. torreyi var. curvata. 9- M. rotata var. rotata. 10- M. radiata var. brasiliensis. 11- M. mahabuleshwarensis var. amazonensis. 12- M. laticips var. acuminata. 13- E. ornans. Escala $10 \mu \mathrm{m}$. 
Micrasterias siolii Scott \& Croasdale, Acta Botanica Fennica, Figura 86,1965. (Figura 6)

Células cerca de 1,1 vezes mais larga que longa; semicélulas 5- lobadas; lobo polar cuneado, com ápice largamente retuso, seus ângulos divididos verticalmente em duas partes. Cada lobo lateral dividido em lóbulos; margem do lobo lateral convexo.

Medidas: 235 X $280 \mu \mathrm{m}$; istmo $35 \mu \mathrm{m}$; ápice $146 \mu \mathrm{m}$.

Ocorrência: EN12 (INPA 223987).

Distribuição geográfica no Amazonas: Lago Tupé (Melo et al., 2005a).

Micrasterias torreyi Bailey var. curvata, Krieger, Ibid, p.98, t. 134:3, 1939. (Figura 8)

Células cerca de 1 vez mais larga que longa; semicélula de contorno semicircular, 5- lobada, incisões interlobares profundas, lobos basais e laterais divididos em lóbulos, 2denticulados.

Medidas: 216 X $224 \mu \mathrm{m}$; istmo $39 \mu \mathrm{m}$; ápice $73 \mu \mathrm{m}$.

Ocorrência: ET9 (INPA 224015).

Distribuição geográfica no Amazonas: Rio Preto da Eva (Thomasson, 1971).

\section{CONSIDERAÇÕES FINAIS}

Foi registrado um total de 12 táxons, sendo cinco do gênero Euastrum e sete do gênero Micrasterias. Embora Micrasterias tenha sido o gênero com maior número de espécies, estas foram registradas em apenas 35\% das amostras analisadas, enquanto Euastrum esteve presente em $90 \%$ da amostras (Figura 14). E. evolutum var. perornatum e E. ornans foram as únicas espécies presentes em todas as estações analisadas.

Apesar de os dois ambientes estudados apresentarem, de um modo geral, propriedades da água semelhantes (Melo et al. 2005b), o maior número de espécies foi registrado no rio Negro, onde foram registradas todas as espécies, exceto M. radiata Hassal var. brasiliensis Grönblad. Já no lago Tupé foi registrado apenas sete espécies, três de Euastrum (E. evolutum var. perornatum; E. ornans e E. sinuosum) e quatro de Micrasterias (M. borgei; M. radiata var. brasiliensis; M. torreyi; M. rotata var. rotata). Esta importância do rio Negro em relação riqueza de espécies de algas foi também registrada por Melo et al. (2005a,b) em relação ao fitoplâncton, em geral, e as desmídias, em especial.

Temporalmente, o período de águas altas (maio-julho) foi o que apresentou menor riqueza de espécies, uma vez que apenas E. evolutum var. perornatum, E. ornans e E. sinuosum foram registradas neste período. Nenhuma espécie de

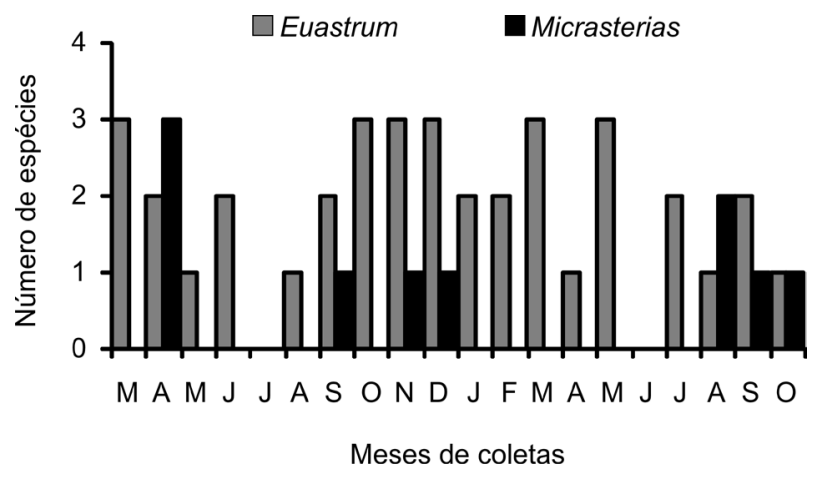

Figura 14 - Variação temporal do número de espécies de Euastrum e Micrasterias no período de março de 2002 a outubro de 2003.

Micrasterias (Figura 14) foi registrada neste período do ciclo hidrológico. Este reduzido número de espécies no período de águas altas pode estar associado à influência do pulso de inundação sobre este grupo de algas, conforme discutido em Melo et al.(2005a).

\section{AGRADECIMENTOS}

Os autores agradecem a Edinaldo Nelson dos Santos-Silva coordenador do Projeto BioTupé, ao CNPq/INPA pelas bolsas de PIBIC concedidas ao segundo e terceiro autor, à CAPES pela bolsa e auxílio PRODOC concedida ao primeiro autor e ao CNPq pelo auxílio financeiro Proc. 473699/2004-4.

\section{BIBLIOGRIA CITADA}

Bicudo, C.E.M.; Bicudo R.M.T. 1970. Algas de águas continentais brasileiras: chave ilustrada para identificação de gêneros. Editora Universidade de São Paulo, São Paulo. 228pp.

Coesel, P.F.M. 1982. Structural characteristics and adaptations of desmids communities. J. Ecol., 70: 163-177.

Förster, K. 1964. Desmidiaceen aus Brasilienz. Teil: Bahia, Goyaz, Piauhy und Nord-Brasilien. Hydrobiologia, 23(3/4): 321-505.

Förster, K. 1969. Amazonische Desmidieen. 1. Areal Santarém. Amazoniana, 2: 5-232.

Förster, K. 1974. Amazonische Desmidieen. 2. Areal Maués-n Abacaxis. Amazoniana, 5(2): 135-242.

Hoek, C., Mann, D.G.; Jahns, H.M. 1997. Algae: an introduction of phycology. Cambridge University Press, Cambridge. 627pp.

Lopes, M.R.M.; Bicudo, C.E.M. 2003. Desmidioflórula de um lago da planície de inundação do rio Acre, Estado do Amazonas, Brasil. Acta Amazônica, 33(2): 167-212.

Martins, D.V. 1986. Gêneros Euastrum Ehrenberg, Micrasterias Agardh e Triploceras Bailey (Desmidiaceae) dos lagos Cristalino e São Sebastião, Estado do Amazonas, Brasil. Universitas (Ciência), 38: 43-58. 
McCourt, R. M.; Karol, K. G.; Bell, J.; Helm-Bychowski, K. M.; Grajewska, A.; Wojciechowski, M. F.; Hoshaw, R. W. 2000. Phylogeny of the conjugating green algae (Zygnemophyceae) based on rbcL sequences. J. Phycol. 36:747-58.

Melo, S.; Sophia, M.G.; Menezes M.; Souza, C. A. 2004. Biodiversidade de algas planctônicas do Parque Nacional do Jaú: Janela Seringalzinho. In: Borges, S. H.; Iwanaga, S.; Durigan, C. C.; Pinheiro, M. R.(Eds). Janelas para a biodiversidade do Parque Nacional do Jaú: uma estratégia para o estudo da biodiversidade da Amazônia. Ed. Ipiranga, Brasília-DF. p.83-95.

Melo, S.; Rebelo S.R.M.; Souza, K.F.; Soares, C.C.; Sophia, M.G .2005a. Desmídias com ocorrência planctônica. In: Santos Silva, E.N.; Aprile, F.M.; Scudeller, V.V.; Melo, S. (Eds). Biotupé: meio físico, diversidade biológica e sócio-cultura do baixo rio Negro, Amazônia Central. Manaus. p. 99- 108.

Melo, S.; Rebelo S.R.M.; Souza, K.F.; Soares, C.C.; Sophia, M.G .2005b. Fitoplâncton ocorrência planctônica. In: Santos Silva, E.N.; Aprile, F.M.; Scudeller, V.V.; Melo, S. Biotupé: meio fisico, diversidade biológica e sócio-cultural do baixo rio Negro, Amazônia Central. Manaus. p. 87-98.
Mix, M. 1972. Die feinstruktur der zellwände bei Mesotaeniaceae und Gonatozygaceae mit einer vergleienden Betrachung der ver vergleienden Wandtypen der Conjugatophytceae und über deren systematischen Wert. Arch. Mikrobiol., 81: 197-220.

Prescott, G.W.; Croasdale, H.T.; Vinyard, W.C. 1977. A synopsis of North American Desmids, II. Desmidiaceae: Placodermae, Sec. 2. Lincoln and London Ed. 413pp.

Růžička, J. 1981. Die Desmidiaceen Mitteleuropas. Band 1, 2. Lief, 293-736 pp. E. Schweizerbart'sche Verlagsbuchhandlung, Stuttgart.

Sioli, H. 1950. Das Wasser im amazonasgebiet. Fortschr., 26: 274280.

Thomasson, K. 1971. Amazonian algae. Mém. Inst. r. Sci. nat. Belg., Sér10, 86: 1-57.

Uherkovich, G. 1981. Algen aus einigen Gewaessern Amazoniens. Amazoniana, 7(2): 191-219.

Uherkovich, G.; Rai, H. 1979. Algen aus Rio Negro und seinen Nebenflüssen. Amazoniana VI, (4):611-638.

Recebido em 31/07/2007

Aceito em 18/12/2008 
